\title{
ESQUEMAS CONCEPTUALES DE LOS ALUMNOS SOBRE LA RESPIRACIÓN
}

\author{
BANET, E. y NÚNEZZ, F. \\ Escuela Universitaria de Magisterio. C.P. San Isidoro. Murcia.
}

Este trabajo fue presentado en los IX Encuentros de Didáctica de las Ciencias Experimentales (Tarragona, Septiembre de 1988), y forma parte del proyecto de investigación financiado por la DGICYT de número de referencia PB87-0683.

\begin{abstract}
SUMMARY
In this article we report our findings about the ideas and beliefs sustained by a sample of students (from a variety of levels, i.e. EGB 6th and 8th grade levels as well as School of Education students) on the topic of human breathing. As well as specific misconceptions, alternative frameworks to scientific knowledge, which should be taken into account by the teacher when planning a lesson, are pointed out.
\end{abstract}

\section{INTRODUCCIÓN}

A lo Iargo de los últimos años, se ha puesto de manifiesto que el aprendizaje de los conceptos científicos se encuentra mediatizado por los conocimientos previos que poseen los alumnos. Estas ideas, estructuradas en forma de esquemas conceptuales, más o menos coherentes, contienen frecuentemente errores o imprecisiones, de diferente grado de importancia, persistentes y, por tanto, de difícil modificación (Giordan 1982, Gil 1986). Por eilo, como señala Driver (1986), estas nociones deben constituir un punto de referencia importante, al planificar las tareas de aprendizaje.

Los resultados que presentamos a continuación, completan investigaciones anteriores (Bazan 1984, Giordan 1987), y constituyen un paso más dentro de nuestro proyecto de trabajo, orientado a realizar una propuesta didáctica sobre nutrición humana para el Ciclo Superior de EGB (Banet y Núñez, 1988, 1989), que tenga en cuenta las representaciones de los alumnos.

\section{MÉTODOS DE TRABAJO}

A partir de un mapa de conceptos (Novak y Gowin, 1988), en el que intentamos poner de manifiesto Ios conceptos más relevantes relacionados con la respira- ción, y establecer las relaciones más significativas existentes entre ellos, preparamos los guiones de entrevistas individuales, así como los cuestionarios, de acuerdo con la secuenciación recogiđa en el cuadro 1.

\section{Cuadro I}

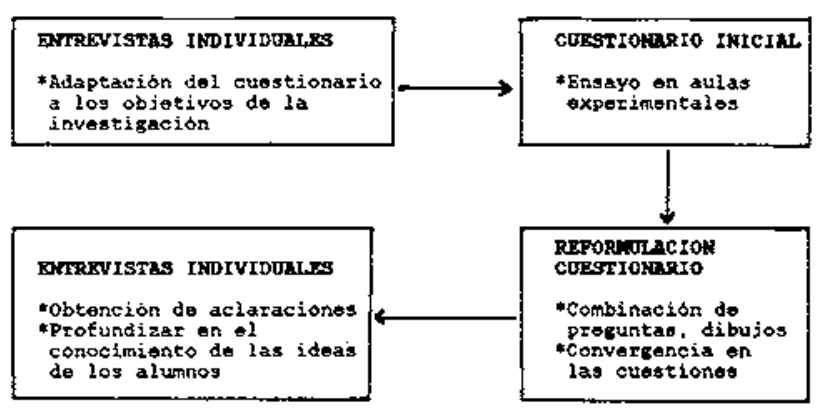

Los procedimientos de exploración han tenido en cuenta las consideraciones realizadas en un trabajo anterior (Banet y Núnez, 1988), y se han centrado tanto en los cuestionarios como en las entrevistas individuales sobre Ios siguientes aspectos: 
A) Para conocer sus ideas sobre la anatomía del aparato respiratorio, les propusimos las siguientes preguntas:

1. Dibuja el aparato respiratorio sobre la silueta que te presentamos, indicando el nombre de cada uno de los organos que forman parte del mismo.

2. De los siguientes órganos, señala con una cruz los que forman parte del aparato respiratorio:

$\begin{array}{llll}\text { Pulmones } & \text { Corazón } & \text { Es6fago } & \text { Laringe } \\ \text { Boca } & \text { Alveolos } & \text { Tráquea } & \text { Ventrículos } \\ \text { Bronquios } & \text { Faringe } & \text { Fosas nasales } & \text { Bronquiolos }\end{array}$

3. Según el recorrido del aire, ordena los órganos que has señalado en la cuestión anterior (procura no olvidar ninguno):

a. Boca o fosas nasales.

b.

c.

etc.

\section{Pulmones}

Aunque en cierto modo se trata de cuestiones que requieren del alumno una repetición en sus respuestas, nos parece necesario, al menos en los niveles básicos, recoger esta información. El análisis de conjunto de las respuestas a estas preguntas nos permitirán conocer suficientemente las ideas de los alumnos al respecto. A partir de octavo de EGB, podríamos añadir una cuestión adicional relacionado con la estructura interna de Ios pulmones:

4. La siguiente silueta corresponde a uno de los pulmones.

Dibuja cómo es por dentro, señalando el camino que sigue el aire en su interior.

B) Se formularon, también, diversas preguntas cuyo objetivo era poder apreciar, entre otros aspectos, las nociones de los alumnos respecto a las consecuencias del proceso de ventilación pulmonar:

Comenzamos por intentar conocer sus ideas respecto a la composición del aire atmosférico con las siguientes preguntas:

5) El aire atmosférico contiene (Señala la que te parezca más correcta):
solo aire
aire y oxígeno
aire y nitrógeno
aire, oxígeno y dióxido
aire y dióxido de carbono
oxígeno, dióxido de
carbono y nitrógeno

$¿$ Conoces alguna otra sustancia que esté contenida en el aire?

6) La cantidad de oxígeno que contiene el aire atmosférico es:

El aire atmosférico no contiene oxígeno.

Poca cantidad (menos del 25\%).

Regular cantidad (entre el 25 y $50 \%$ ).
Bastante cantidad (entre el 50 y $75 \%$ ).

El aire atmosférico tiene muchísimo oxígeno (más del $75 \%$ ).

¿Podrías precisar aproximadamente qué cantidad contiene?

7) La cantidad de dióxido de carbono que contiene el aire atmosférico es:

El aire atmosférico no contiene dióxido de carbono.

Contiene muy poca cantidad (menor del $5 \%$ ).

Contiene poca cantidad (entre el 5 y $25 \%$ ).

Regular cantidad (entre 25 y $50 \%$ ).

Bastante cantidad (entre 50 y $75 \%$ ).

El aire atmosférico contiene muchísimo dióxido de carbono (más del 75\%).

¿Podrías precisar aproximadamente qué cantidad contiene?

Estas preguntas fueron, por razones obvias, suprimidas en los cuestionarios empleados en sexto de EGB y, en cualquier caso, las respuestas se analizaron con ciertas reservas en octavo (aunque antes se había consultado al profesor sobre la dificuitad de los alumnos para poder responder a las mismas).

A continuación, abordamos de un modo más directo el problema con las siguientes cuestiones (asegurándonos de que en cada caso los alumnos de los niveles básicos comprendían el significado de "dióxido de carbono", al menos desde el punto de vista del intercambio de gases durante la ventilación pulmonar):

8) El aire que tomamos del exterior lleva:

\begin{tabular}{|c|c|}
\hline $\begin{array}{l}\text { Hidrógeno } \\
\text { Oxígeno } \\
\text { Monoxido de carbono }\end{array}$ & $\begin{array}{l}\text { Nitrógeno } \\
\text { Dióxido de carbono } \\
\text { Vapor de agua }\end{array}$ \\
\hline $\begin{array}{l}\text { 9) El aire que expulsamos } \\
\text { Hidrogeno } \\
\text { Oxígeno } \\
\text { Monóxido de carbono }\end{array}$ & $\begin{array}{l}\text { lieva: } \\
\text { Nitrógeno } \\
\text { Dióxido de carbono } \\
\text { Vapor de agua }\end{array}$ \\
\hline
\end{tabular}

En Ios niveles básicos, nuestra atención se dirigió de forma casi exclusiva a comprobar el balance $\mathrm{O}_{2} / \mathrm{CO}_{2}$ en Ia inspiración/espiración (se confunde hidrógeno con nitrógeno, ¿monóxido de carbono?... ¿gases?).

C) Por último, era necesario abordar los aspectos relacionados con el transporte de gases por la sangre y la respiración celular, lo cual no resulta una tarea fácil cuando se estudian niveles educativos tan diferentes. Es evidente la necesidad de adaptar las preguntas a cada nivel y apoyarse bastante en las entrevistas individuales.

En las entrevistas indagamos sobre:

Destino del oxígeno que contiene el aire inspirado. Procedencia del dióxido de carbono que contiene el aire que expulsamos durante la espiración.

Necesidad y utilización del oxígeno por las células. Respiración celular (sustancias que intervienen y resultados). 
Los cuestionarios inciuirían las siguientes preguntas:

10) Indica si son verdaderas (V) o falsas (F) las siguientes frases:

El oxígeno se transporta por la sangre de los pulmones a la sangre.

El oxígeno se transporta por la sangre de las células a los pulmones.

El oxígeno no se transporta por la sangre, se queda en los pulmones.

El díxido de carbono se transporta por la sangre de los pulmones a las células.

El díxido de carbono se transporta por la sangre de las células a los pulmones.

El díxido de carbono no puede ser transportado por la sangre porque es perjudicial.

Las células recogen oxígeno de la sangre.

Las células no toman ni recogen oxígeno de la sangre. Las células no toman ní recogen dióxido de carbono de la sangre.

11) Indica si son verdaderas (V) o falsas (F) las siguientes frases:

Todas las células del cuerpo necesitan oxígeno

AIgunas células pueden no necesitar oxígeno.

Los huesos no necesitan oxígeno.

Los huesos necesitan oxígeno.

Las células đel cuerpo producen dióxido de carbono.

Las células del cuerpo no producen dióxido de carbono.

El aire que expulsamos contiene casi exclusivamente dioxido de carbono.

El aire que expulsamos contiene otros gases en proporciones elevadas.

Finalmente, y aun reconociendo la dificultad para responder a esta pregunta por parte de alumnos de Ciclo Superior de EGB, decidimos formular en Ios 3 niveles la siguiente pregunta:
13) Explica para qué se utiliza el oxígeno. ¿Qué sustancias reaccionan con el oxígeno en las células (Señala 3 como máximo):
La materia viva

Las sustancias necesarias

Los alimentos

Las proteínas
Los hidratos de carbono

Las grasas

Los productos de

combustión
¿Qué se produce como resultado de esas reacciones? (Señala todas las posibilidades que consideres adecuadas):

$\begin{array}{ll}\text { Sustancias de desecho } & \text { Agua } \\ \text { Dibxico de carbono } & \text { Materia orgánica } \\ \text { Proteínas } & \text { Vitaminas }\end{array}$

En la Tabla I, presentamos las características correspondientes a la población escolar que ha servido de base a nuestro estudio.

\begin{tabular}{|c|c|c|c|c|}
\hline \multicolumn{5}{|c|}{$\begin{array}{c}\text { Tabla I } \\
\text { Población explorad }\end{array}$} \\
\hline NIYEL & CURSO & ALUMNOS & CENTROS & CONDICIONES \\
\hline$E G B$ & $6^{2}$ & 150 & 4 & Pre-test \\
\hline EGB & $8^{2}$ & 120 & 4 & Pre-test \\
\hline Magisterio* & $3^{\circ}$ & so & 1 & Pre-test \\
\hline
\end{tabular}

\section{RESULTADOS Y DISCUSIÓN}

\section{Anatomia del aparato respiratorio}

De los resultados obtenidos, destacamos como más significativos los siguientes: Algo menos de la mitad de

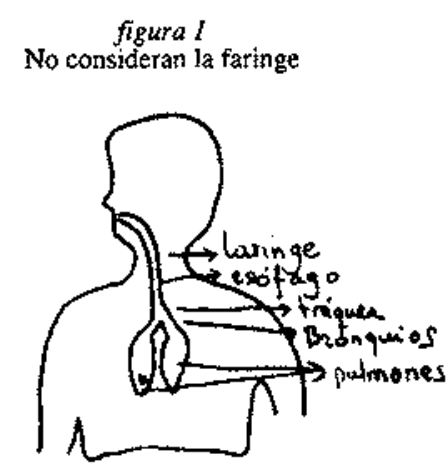

(\%)

$6^{\circ} \mathrm{EGB}$

$8^{\circ} \mathrm{EGB}$

EUM figura 2

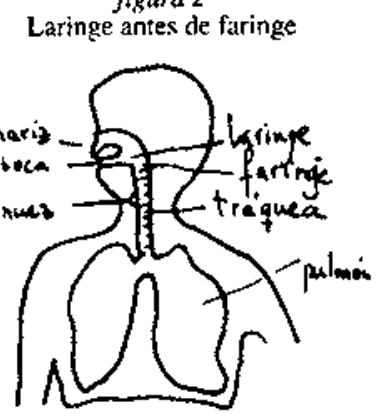

(\%) 
alumnos conocen todos los órganos que forman parte del aparato respiratorio y el orden en el que están situados. Su dibujo no presenta especiales dificultades, no constatando las observaciones de Bazan (1984), que pone de manifiesto dibujos que comunican el corazón con este sistema. Se observa, sin embargo, un profundo desconocimiento de la estructura interna de los pulmones (figura 3), ignorándose la relación entre las vías respiratorias y los alveolos pulmonares.

Son errores frecuentes, los relativos al trayecto bocalaringe (figuras 1 y 2), por el desconocimiento de las relaciones que, a nivel anatómico, existen entre los aparatos digestivo y respiratorio (Banet y Núñez 1988).

\section{Composición del aire atmosférico, inspirado y espi- rado}

Este aspecto, constituye un punto de referencia importante para establecer los esquemas conceptuales que poseen los alumnos sobre la respiración. La referencia al aire atmosférico fue necesaria al comprobar, en entrevistas previas, como algunos establecían diferencias entre su composición y la del aire inspirado.

Responder a cuestiones de esta naturaleza puede suponer dificultades para alumnos de niveles básicos (términos como nitrógeno o hidrógeno les resultan confusos o desconocidos). Por esto, en la Tabla II, presentamos únicamente los resultados correspondientes al oxígeno y al dióxido de carbono.

\begin{tabular}{|c|c|c|c|}
\hline \multicolumn{4}{|c|}{$\begin{array}{l}\text { Tabla II } \\
\text { Ideas de los alumnos sobre la composicion del aire } \\
\text { (resultados expresados en \%) }\end{array}$} \\
\hline Aire atmosférice & $6^{\circ} \mathrm{ECB}$ & $8^{\circ} \mathrm{EGB}$ & EUM \\
\hline Contiene $\mathrm{O}_{2}$ y $\mathrm{CO}_{2}$ & 84 & 83 & 92 \\
\hline Sola contiene 02 & - & - & - \\
\hline Sólo contiene $\mathrm{CO}_{2}$ & - & - & . \\
\hline \multicolumn{4}{|l|}{ Aixe inspirado } \\
\hline Contiene $\mathrm{O}_{2}$ y $\mathrm{CO}_{2}$ & 74 & 81 & 71 \\
\hline Solo contiene 02 & 22 & 19 & 23 \\
\hline \multicolumn{4}{|l|}{ Aire espirado } \\
\hline Contiene $\mathrm{O}_{2}$ y $\mathrm{CO}_{2}$ & 15 & 38 & $4 i$ \\
\hline
\end{tabular}

Una primera consideración se desprende de estos resultados: Mientras que uno de cada cuatro o cinco alumnos piensan que el aire inspirado está formado solamente por oxígeno, ninguno indica que el aire atmosférico contenga exclusivamente este gas (más del $80 \%$ de los alumnos de niveles básicos, y casi todos los de Magisterio, reconocen, al menos, la presencia de dióxido de carbono). Elio pone de manifiesto, como hemos podido confirmar en entrevistas individuales, que la inspiración conlleva un proceso de selección de gases, de manera que a los pulmones sólo llega oxígeno (Categotía 1, Tabla III).

P. ¿En qué consiste la respiración?; R. "En tomar oxígeno del aire y expulsar el dioxido de carbono"; "En inspirar oxígeno para que vaya a los pulmones".
Tabla III

Modelos sobre la respiracion

\begin{tabular}{|c|c|c|c|}
\hline $\begin{array}{l}\text { VENT ILACION } \\
\text { PULMONAR } \\
\text { GASES } \\
\text { RESPIRATORIOS }\end{array}$ & $\begin{array}{l}\text { CATEGORIA I } \\
\text { Entra O=, se } \\
\text { expulsz COO }\end{array}$ & $\begin{array}{l}\text { CATEgORIA } \\
\text { Entra aire ( }) \\
\text { se expulsa } \\
\text { Com (b) }\end{array}$ & $\begin{array}{l}\text { CATEGQRIA } 3 \\
\text { Entra dire } \\
\text { bIt, se ex- } \\
\text { Dulse dire. }\end{array}$ \\
\hline $\begin{array}{l}\text { CATEGORIA A: } \\
\text { *O a pulmones } \\
\text { * Se expulsa el } \\
\text { CO inspirado }\end{array}$ & BODELC I & & \\
\hline $\begin{array}{l}\text { CATEGORIA B: } \\
\text { *OL a celulas } \\
\text { dande es uti- } \\
\text { lizado } \\
\text {-Se expulsa el } \\
\text { CO inspirado }\end{array}$ & MODELLO Z & ODELO 3 & \\
\hline $\begin{array}{l}\text { CATEGORIA C: } \\
\text { 40, a Celulas } \\
\text { donde es uti- } \\
\text { li } 2 \text { ado. } \\
\text { - Se obtiene } \\
\text { Energia } \mathrm{CO} \text {. }\end{array}$ & & WODELO 4 & MGDELO 5 \\
\hline
\end{tabular}

Por otra parte, aunque tres de cada cuatro alumnos reconocen la presencia de varios gases en el aire espirado (fundamentalmente dióxido de carbono $y$, en ocasiones, vapor de agua, nitrógeno o hidrógeno), un número muy elevado de ellos indican que este aire no contiene oxígeno ( $\mathrm{i}$ La respiración consiste, precisamente, en introđucir y no en expulsar este gas! Categoría 2, Tabla III).

Por último, en un tercer grupo incluimos aquéllos que opinan que tanto el aire inspirado como el espirado, estấn compuestos por diversos gases (Categoría 3. Tabla IV). Aproximadamente, uno de cada tres alumnos de octavo de EGB y Magisterio responden en este sentido (en sexto tan sólo un 10\%).

\section{La respiración a nivel celular}

Casi todos los alumnos (salvo algunas excepciones en EGB) reconocen "Ias células", "los tejidos"... como destino del oxígeno que respiramos. Sin embargo, en algunos casos se señala que no todas las células lo necesitan, y la mayoría de los alumnos de niveles básicos afirman que los huesos no reciben oxígeno. No obstante, de acuerdo con Bazan (1984), en ocasiones (aunque hay que reconocer que con poca frecuencia) se restringe la respiración a un fenómeno pulmonar (Categoría A, Tabla IV). Sólo en Magisterio se observa claramente la relación del oxígeno con procesos de combustión celular, metabolismo...

AI preguntar por las sustancias que "se queman", muchos alumnos de EGB no responden, $y$ los que lo hacen, emplean términos ambiguos. En todo caso, cuando existen referencias a sustancias concretas, se mencionan fundamentalmente las proteínas (incluso en Magisterio).

Por último, muchos alumnos señalan, en algún momento, la energía, cuando se valora la utilidad del oxígeno que respiramos, pero sólo en Magisterio se reconoce el dióxido de carbono como producto resultante 
de las combustiones celulares. Para muchos alumnos, el díxido de carbono que eliminamos en la espiración es el que habia entrado en la inspiración (Categoría B. Tabla III). Completamos esta Tabla con otra categoría (C), en la que se incluyen aquéllos que indican, como consecuencia del metabolismo celular, la produccion de energía y de dióxido de carbono. Este gas será transportado por la sangre, para ser expulsado al exterior.

\section{MODELOS SOBRE LA RESPIRACIÓN}

Los resultados obtenidos nos permiten establecer, a modo de conclusión, los siguientes modelos que responden a esquemas conceptuales de los alumnos (Tabla IV), aunque somos conscientes de que dicha síntesis oculta otros intermedios, diff́ciles de clasificar. En la figura 4, presentamos la incidencia que podría corresponder a cada uno de ellos en los diferentes niveles.

Si bien los modelos 1 y 2 tienen escasa incidencia, hacemos referencia a ellos para dejar constancia de su existencia (la respiración consiste en la entrada de oxígeno a los pulmones y la salida de díxxido de carbono), y llamar la atención sobre los errores a los que puede inducir ciertas definiciones que aparecen en los libros de texto.

El modelo 3, el más frecuente en sex to de EGB, predomina la idea de que el aire espirado carece de oxígeno. Algunos alumnos de este nivel pueden considerarse dentro del modelo 4 , si bien responden de forma confusa o contradictoria cuando se les pregunta, por ejemplo, cómo producen las células el dióxido de carbono.

Aunque no ha sido nuestra intención realizar una comparación rigurosa entre niveles educativos, es evi-

\section{REFERENCIAS BIBLIOGRÁFICAS}

BANET, E. y NÚÑEZ, F., 1988. Ideas de Ios alumnos sobre la digestión: Aspectos anatómicos, Enseñanza de las Ciencias, Vol. 6(1), pp. 30-37.

BANET, E. y NÚÑEZ, F., 1989. Ideas de los alumnos sobre la digestion: Aspectos fístológicos, Enseñanza de las Ciencias, vol. 7(1), pp. 35-44. dente que los efectos de la instrucción deben producir un desplazamiento hacia los modelos 4 y 5 . No obstante, parece interesante resaltar que muchos de los alumnos incluidos en el último modelo: a) Desconocen la naturaleza de las sustancias que intervienen en la respiración celular e ignoran los detalles más elementales de este proceso; b) Consideran la proporción de oxígeno del aire espirado escasa, jdesde luego muy inferior a la de dióxido de carbono!, que sería muy elevada.

\section{ALGUNAS CONSIDERACIONES DIDÁCTICAS}

El estudio de las representaciones de los alumnos no sirve de mucho si de sus resultados no se derivan consecuencias que orienten las tareas de clase del profesor. En este sentido, dentro de los planteamientos didácticos que estamos desarrollando sobre nutrición hurnana en octavo de EGB, basados en los conocimientos previos de los alumnos, y en lo que a la respiración se refiere, ponemos de manifiesto las siguientes consideraciones:

A nivel anatómico, resulta útil establecer las relaciones existentes entre los aparatos digestivo y respiratorio, así como analizar la estructura alveolar de los pulmones y su relación con las vías respiratorias.

Es preciso diferenciar la ventilación pulmonar de los intercambios gaseosos a nivel de alveolos, haciendo referencia a la composición de los aires atmosférico, ins. pirado y espirado.

Por último, y aun teniendo en cuenta el nivel de los alumnos, conviene estudiar aspectos relativos al trans. porte de gases por la sangre, los intercambios a nivel de los tejidos, y la utilización del oxígeno a nivel celular.
BAZAN, M., 1984. Les hypothèses sur la mécanique respiratoire au niveau de la classe de sixième. Actas sixièmes Journés Internationales sur l'éducation scientifique, pp. $729-735$.

DRIVER, R., 1986. Psicología cognoscitiva y esquemas conceptuales de los alumnos, Enseñanza de las Ciencias, vol. $4(1)$, pp. $3+15$. 
GIL, D., 1986. La metodología científica y la enseñanza de las ciencias. Unas relaciones controvertidas, Enseñanza de las Ciencias, Vol. 4(2), pp. 111-121.

GIORDAN, A., 1982. La enseñanza de las Ciencias. (Pablo del Rio: Madrid).

GIORDAN, A., 1987. Des modeles personnels et historiques à propos du champ conceptuel de respiration à leurs utilisations pédagogiques. II Congreso Internacional sobre investigación en la didáctica de las Ciencias y de las Matemáticas. Valencia, 23-25 de Septiembre.

NOVAK, J.D. y GOWIN, D.B., 1988. Aprendiendo a aprender. (Martínez Roca: Barcelona). 